Military Technical College Kobry Elkobbah, Cairo,

Egypt.

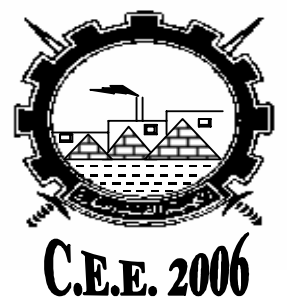

$3^{\text {rd }}$ International Conference

On

Chemical \& Environmental Engineering

\title{
CYANOACETANILIDES IN HETEROCYCLIC SYNTHESIS (PART IV): A CONVENIENT ONE-STEP SYNTHESIS OF 2-IMINO CHROMENE-3- CARBOXAMIDE, CHROMENONE, BENZOCHROMENE, CHROMENOCOUMARIN AND CHROMENOPYRIDINE DERIVATIVES FOR ANTIMICROBIAL EVALUATION
}

\author{
El-Sharief A. M. *, El-Sehemi A.G. **, Aly M. M. ", Salem M. A. *, \\ Mohamed Y. A. ${ }^{*}$ and Ammar Y. A.
}

\begin{abstract}
Cyanoacetanilides $(1 a, b)$ were reacted with o-hydroxyaldehydes under different conditions. Thus, cyclocondensation of $1 \mathrm{a}, \mathrm{b}$ with salicylaldehyde, 2-hydroxy-1naphth-aldehyde and 7-hydroxy-5-methoxycoumarin-6-carboxaldehyde in ethanolic amm. acetate afforded the corresponding 2-iminochromene derivatives 3,7 and 9 respectively. On the other hand, repeating the same reaction in presence of $\mathrm{Ac}_{2} \mathrm{O} / \mathrm{AcONa}$, the corresponding chromen-2-one derivatives 4,8 and 10 were obtained. Compounds 4,8 and 10 were also synthesized through the hydrolysis of 2imino derivatives using $\mathrm{EtOH} / \mathrm{HCl}$. A number of chromeno[3,4-c]-pyridine derivatives $11,13,14$ and 16 were prepared from the reaction of 2-iminochromenes with malononitrile, ethyl cyanoacetate, and cyanoacetanilide. Some of these compounds were screened in vitro for their antimicrobial activities.
\end{abstract}

\section{KEYWORDS}

Chromenone, Benzochromene, Chromenocoumarin, Chromeno-pyridine, Antimicrobial Activity

\footnotetext{
Faculty of Science, Al-Azhar University.

${ }^{\star *}$ Chemistry Department, Teacher s Colleges, Abha Saudia.
} 


\section{INTRODUCTION}

It is well known that antibacterial ${ }^{1}$, coronary dilatory ${ }^{2}$ and hypothermal agents ${ }^{3}$ as well as potential laser dyes ${ }^{4}$ have been reported for many chromene derivatives. Moreover, coumarin derivatives are well known to have anticoagulant and antibacterial activities ${ }^{5-9}$. Furthermore, benzocoumarins are of great biological importance due to their analgesic and antihypertensive activities ${ }^{10}$. The present study is part of our program aimed at developing easy routes for the synthesis of fused heterocyclic compounds starting with cyanoacetanilides as simple organic compounds. Also, we report here the synthesis of chromene and coumarin carboxamide derivatives and their utility as building blocks in the synthesis of novel pyridochromene derivatives to evaluate their antimicrobial activity.

\section{RESULTS AND DISCUSSION}

Condensation of cyanoacetanilides $(\mathbf{1} \mathbf{a}, \mathbf{b})^{11}$ with salicylaldehyde $(\mathbf{2})$ in ethanolic amm. acetate afforded 2-imino-3-(N-substituted-phenylcarboxamido)-2H-chromenes $(3 a, b)$ as colored solids in high yield, (Scheme1).

The structure of compounds (3a,b) were established by elemental analysis and spectral data. IR spectrum of compound (3a) showed absorption bands at 3403 , $3316(\mathrm{NH})$ and $1681 \mathrm{~cm}^{-1}(\mathrm{C}=\mathrm{O}) .{ }^{1} \mathrm{H}-\mathrm{NMR}$ spectrum of (3a), (ס, ppm) (DMSO-d 6$) 2.2$ $\left(\mathrm{s}, 3 \mathrm{H}, \mathrm{CH}_{3}\right), 6.8-7.8(\mathrm{~m}, 8 \mathrm{H}, \mathrm{Ar}-\mathrm{H}), 8.5(\mathrm{~s}, 1 \mathrm{H}, \mathrm{H}-4)$. The mass spectra of $(3 \mathrm{a}, \mathrm{b})$ display the correct molecular ions expected for the molecular formula. The fragmentation patterns are in accordance with the assigned structure. The primary cleavage of the molecular ion occurs at the amide bond giving rise to the acylium ion $\mathrm{m} / \mathrm{z} 172$.

On the other hand, interaction of $(\mathbf{1} \mathbf{a}, \mathbf{b})$ with salicylaldehyde in the presence of $\mathrm{Ac}_{2} \mathrm{O} / \mathrm{AcONa}$, gave 3-(N-substituted-phenylcarboxamido)chromen-2-ones $(\mathbf{4 a} \mathbf{a}, \mathbf{b})$ in reasonably good yield. IR spectrum of compound (4a) showed absorption bands at $3271(\mathrm{NH})$ and $1705 \mathrm{~cm}^{-1}$ (CO-lactone). ${ }^{1} \mathrm{H}-\mathrm{NMR}$ spectrum (DMSO-d $\mathrm{d}_{6}$ ) of compound (4b) revealed multiplet at $7.4-8.0 \mathrm{ppm}$ for aromatic protons, singlet at $8.8 \mathrm{ppm}$ assigned to chromene- $\mathrm{H}$ and singlet at $10.6 \mathrm{ppm}$ for $\mathrm{NH}$. Mass spectrum of compound (4b) showed a molecular ion peak at $299(25 \%)$ with a base peak at $\mathrm{m} / \mathrm{z}=$ $173\left(\mathrm{M}-\mathrm{Cl} . \mathrm{C}_{6} \mathrm{H}_{4} \mathrm{NH}\right)$. Other significant peaks were appeared at m/z: $101(23.9 \%), 89$ $(22 \%)$ and $51(7.9 \%)$. The structure of 4 was further confirmed through their synthesis upon hydrolysis of $\mathbf{3}$ with $\mathrm{EtOH} / \mathrm{HCl}$ or acetic acid.

Similarly, compounds $1 \mathbf{a}$ or $\mathbf{b}$ were reacted with 2-hydroxy-1-naphthaldehyde (5) and 7-hydroxy-5-methoxycoumarin-6-carboxaldehyde (6) under the same conditions of

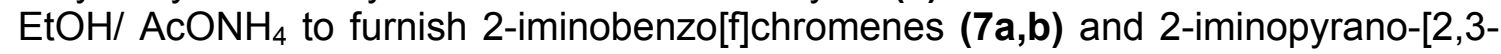
g] coumarins (9) in high yield. While, benzocoumarins $(\mathbf{8 a}, \mathbf{b})$ and pyrano[2,3g]coumarin (10) were synthesized via interaction of (1) with (5) and (6) in the presence of acetic anhydride and sodium acetate. Compounds (8) and (10) were also obtained upon the treatment of compounds (7) and (9) with ethanol containing hydrochloric acid or acetic acid. (Scheme 1). 
The suggested structures (7), (8), (9) and (10) were established on the basis of both analytical and spectral data. IR spectrum of the synthesized compounds (7a) showed vNH vCH-aliph. $v C=\mathrm{O}$ at $3324,329829251684 \mathrm{~cm}^{-1}$ respectively. ${ }^{1} \mathrm{HNMR}$ spectrum of 9 in DMSO- $\mathrm{d}_{6} 4.0\left(\mathrm{~s}, 3 \mathrm{H}, \mathrm{OCH}_{3}\right), 6.42(\mathrm{~d}, 1 \mathrm{H}, \mathrm{H}-6), 7.05-7.52(\mathrm{~m}, 5 \mathrm{H}, \mathrm{Ar}-\mathrm{H})$, 8.1(d,1H, H-7), 8.5(s,1H,H-4) and 9.5, $12.8(2 \mathrm{~s}, 2 \mathrm{H}, 2 \mathrm{NH})$. The mass spectrum of 9 afforded m/z: $396(19.1 \%), 398(\mathrm{M}+2,6.8 \%), 361(\mathrm{M}-\mathrm{Cl} ; 100 \%), 270\left(\mathrm{M}-\mathrm{Cl} . \mathrm{C}_{6} \mathrm{H}_{4} \mathrm{NH}\right.$; $75.2 \%), 227$ [M-(Cl. ${ }_{6} \mathrm{H}_{4} \mathrm{NH}$ and $\left.\mathrm{NHCO}\right) ; 29.8 \%$ ], 199 [M-(Cl. ${ }_{6} \mathrm{H}_{4} \mathrm{NH}, \mathrm{NHCO}$ and CO); $14.5 \%]$.

Moreover, the resulting chromene derivatives have latent functional substituents which have the potential for further chemical transformations giving new routes for the preparation of condensed chromene with possible biological activity.

Thus, the reaction of 2 -iminochromene derivatives $(3 \mathbf{a}, \mathbf{b})$ with malononitrile and ethyl cyanoacetate in refluxing ethanol containing catalytic amount of amm. acetate for 3 hrs afforded in each case a product with analytical and spectral data in good agreement with chromeno[3,4-c]pyridine derivatives (12 a,b) (Scheme 2).

The structures (12) have been confirmed on the basis of elemental analysis and spectral data. IR spectrum of (12a) showed $\mathrm{NH}_{2} \mathrm{NH}$ at 3439, 3348, CN at 2207 and $\mathrm{CO}$ (amido) at $1650 \mathrm{~cm}^{-1}$. Mass spectrum of (12a) showed a molecular ion peak at $\mathrm{m} / \mathrm{z}$ : $342(3.2 \%)$ with a base peak at $\mathrm{mlz} 300\left[\mathrm{M}-\left(\mathrm{CH}_{3}+\mathrm{HCN}\right)\right]$. Other significant peaks were observed at $\mathrm{m} / \mathrm{z} 272\left[\mathrm{M}-\left(\mathrm{CH}_{3}, \mathrm{HCN}\right.\right.$ and $\left.\left.\mathrm{CO}\right) ; 34.0 \%\right], 256\left[\mathrm{M}-\left(\mathrm{CH}_{3}\right.\right.$ $\mathrm{HCN}, \mathrm{CO}$ and $\left.\mathrm{NH}_{2}\right) ; 3.5 \%$ ] and 230 [M-( $\mathrm{CH}_{3}, \mathrm{HCN}, \mathrm{CO}, \mathrm{NH}_{2}$, and $\left.\mathrm{CN}\right) ; 4.36 \%$ ]. ${ }^{1} \mathrm{HNMR}$ spectrum of $(\mathbf{1 2 b})$ in DMSO- $\mathrm{d}_{6}$ revealed the following signals: $=7.3-7.6(2 \mathrm{~m}$, $8 \mathrm{H}, \mathrm{Ar}-\mathrm{H}), 8.8(\mathrm{~s}, 1 \mathrm{H}, \mathrm{NH}), 9.2(\mathrm{~s}, 1 \mathrm{H}, \mathrm{OH})$. The formation of $(\mathbf{1 2 a}, \mathbf{b})$ is assumed to proceed via the addition of an active methylene group to the double bond of chromene to give the Michael adduct which underwent cyclization to afford dihydrochromenopyridine as intermediate which is oxidized to yield the 5iminochromeno-pyridine derivatives.

On the other hand, the dihydrochromenopyridine derivative (13) was obtained upon treatment of $(\mathbf{3 b})$ with malononitrile in boiling $\mathrm{EtOH} / \mathrm{AcONH}_{4} .{ }^{1} \mathrm{HNMR}$ spectrum of structure 13 in DMSO- $\mathrm{d}_{6}$ showed signals at: $4.71(\mathrm{~d}, 1 \mathrm{H}, \mathrm{H}-4), 4.9(\mathrm{~d}, \mathrm{IH}, \mathrm{H}-3), 7.3-$ $7.6(\mathrm{~m}, 8 \mathrm{H}, \mathrm{Ar}-\mathrm{H}), 7.7\left(\mathrm{~s}, 2 \mathrm{H}, \mathrm{NH}_{2}\right), 8.9(\mathrm{~s}, \mathrm{H}, \mathrm{NH})$.

Also, benzochromenopyridine derivative (14) was obtained through interaction of (7b) with malononitrile under the same condition of $\mathrm{EtOH} / \mathrm{AcONH}_{4}$ (Scheme 3).

The structure of (14) was established for the reaction product based on elemental analysis and spectral data. ${ }^{1} \mathrm{HNMR}$ spectrum of (14) in DMSO- $\mathrm{d}_{6}$ showed the following signals: 7.6-8.6 (m, 10H, Ar-H), 8.9-9.0 (d, 2H, NH

Finally, cyanoacetanilide (1 b) was used as active methylene compound and reacted with 2- iminochromene-3-carboxamide $(15)^{12}$ in 1: 1 molar ratio in the presence of amm. acetate to afford the chromenopyridine derivative (16) (Scheme 3). Its ${ }^{1} \mathrm{H}-\mathrm{NMR}$ in DMSO- $\mathrm{d}_{6}$ exhibited 7.4-7.8 (m, 8H, Ar-H), $9.0\left(\mathrm{~d}, 2 \mathrm{H}, \mathrm{NH}_{2}\right), 10.3-10.5(\mathrm{br}, 3 \mathrm{H}$, $3 \mathrm{NH}$ ). 


\section{Antimicrobial Activity:}

Some of synthesized compounds were tested in Vitro against Gram positive bacteria: Bacillus cereus (NCTC-14579), Gram negative bacteria: Proteus mirabilis (NCTC289) and fungi of Penicililum chrysogenum (NCTC-289) using the agar diffusion technique $^{13}$. A I mg/ml solution in DMSQ was used. The bacteria and fungi were maintained on nutrient agar and Czapek'sDox agar media, respectively. DMSO showed no inhibition zones. The agar media were inoculated with different microorganisms culture tested after $24 \mathrm{hrs}$. of incubation at $30^{\circ} \mathrm{C}$ for bacteria and 48 hrs. of incubation at $28^{\circ} \mathrm{C}$ for fungi, the diameter of inhibition zone $(\mathrm{mm})$ were measured (Table 1). Ampicillin in a concentration $(25 \mu \mathrm{g})$ and Mycostatine $(30 \mu \mathrm{g})$ were used as references for antibacterial and antifungal activities, respectively. The minimal inhibitory concentrations (MIC) of some of the tested compounds was measured by a two fold serial dilution method ${ }^{14}$. All the tested compounds exhibited activity towards the Gram negative bacteria. However, none of them showed activity against Penicillium chryssogenum.

Table 1: Antimicrobial activity of some synthesized compounds and inhibition zones.

\begin{tabular}{cccc}
\hline Compd. & Gram positive bacteria & Gram negative bacteria & Fungi \\
\cline { 2 - 4 } No. & $\begin{array}{c}\text { Bacillus cereus } \\
\text { (NCTC-14579) }\end{array}$ & $\begin{array}{c}\text { Proteus mirabilis } \\
(\text { NCTC-289) }\end{array}$ & $\begin{array}{c}\text { Penicillium } \\
\text { chrysogenum Thom } \\
\text { (NCTC-289) }\end{array}$ \\
\hline 3b & - & ++ & - \\
7b & - & ++ & - \\
9b & - & +++ & - \\
12a & - & ++ & - \\
12b & - & ++ & +++ \\
Standard & ++++ & +++ & \\
\hline
\end{tabular}

Standard = Ampicillin $25 \mu \mathrm{g} / \mathrm{ml}^{-1}$ mycostatine

+ : Less active $(0.2-0.5 \mathrm{~cm}) ; \quad++$ : Moderately active $(0.6-1.4 \mathrm{~cm})$;

+++ : Highly active $(1.5-3.0 \mathrm{~cm}) ; \quad++++$ : Very highly active (over $3.0 \mathrm{~cm}) ; \quad-$ :Inactive Standard: For Gram positive and Gram negative bacteria: Ampicillin $25 \mu \mathrm{g} \mathrm{mL}^{-1}$; for fungi: Mycostatine: $30 \mu \mathrm{g} \mathrm{mL}^{-1}$. 


\section{EXPERIMENTAL}

All melting points reported are uncorrected. IR spectra were measured $(\mathrm{KBr})$ on a Shimadzu IR 200 spectrophotometer $\left(v_{\max }\right.$ in $\left.\mathrm{cm}^{-1}\right)$. ${ }^{1} \mathrm{H}-\mathrm{NMR}$ spectra were recorded on a Varian Gemini NMR spectrometer $(200 \mathrm{MHz})$ using tetramethylsilane as an internal standard (Chemical shifts in $\delta, \mathrm{ppm}$ ). Mass spectra were obtained on GC MsQP 1000 EX mass spectrometer at $70 \mathrm{ev}$. Elemental analyses were carried out at the Microanalytical Center of Cairo University. The characteristic data of the prepared compounds are given in Table (1).

\section{2-Iminochromene derivatives 3, 7 and 9:}

A mixture of $1(0.01 \mathrm{~mol})$, the requisite o-hydroxyaldehyde $(0.01 \mathrm{~mol})$ and amm. acetate $(0.015)$ was refluxed in ethanol $(30 \mathrm{ml})$ for $1 \mathrm{~h}$. The solid product was collected by filtration and recrystallized from the proper solvent to give $(3,7$ and $\mathbf{9}$; Table 1).IR spectrum of compound (3a) showed absorption bands at 3403, 3316 (NH), $2923\left(\mathrm{CH}\right.$-aliph) and $1681 \mathrm{~cm}^{-1}(\mathrm{C}=\mathrm{O})$, (3b) $3309(\mathrm{NH}), 2925(\mathrm{CH}$-aliph.) and $1683 \mathrm{~cm}^{-1}$ (CO), 7a : 3324, $3298(\mathrm{NH}), 2925$ (CH-aliphatic) and 1684 (CO), 7b: 3242 $(\mathrm{NH}), 2924(\mathrm{CH}$-aliphatic) and $1671(\mathrm{CO}) .9: 3325(\mathrm{NH}), 2970$ (CH-aliphatic) 1735 (CO- lactone). ${ }^{1} \mathrm{H}-\mathrm{NM} R$ spectra in DMSO-d 6 3a: $2.2\left(\mathrm{~s}, 3 \mathrm{H}, \mathrm{CH}_{3}\right), 6.8-7.8(\mathrm{~m}, 8 \mathrm{H}, \mathrm{Ar}-$ H), $8.5(\mathrm{~s}, 1 \mathrm{H}, \mathrm{H}-4), 9.1,10.6(2 \mathrm{~s}, 2 \mathrm{H}, 2 \mathrm{NH}) .3 \mathrm{~b}: 7.2-7.8(\mathrm{~m}, 8 \mathrm{H}, \mathrm{Ar}-\mathrm{H}), 8.5(\mathrm{~s}, 1 \mathrm{H}, \mathrm{H}-$ 4), 9.1, $12.9(2 \mathrm{~s}, 2 \mathrm{H}, 2 \mathrm{NH}), 7 \mathrm{a}: 2.3\left(\mathrm{~s}, 3 \mathrm{H}, \mathrm{CH}_{3}\right), 7.1-8.2(\mathrm{~m}, 10 \mathrm{H}, \mathrm{Ar}-\mathrm{H}), 8.4(\mathrm{~S}, 1 \mathrm{H}$, chromene-H-4), 9.2, $12.4(2 \mathrm{~s}, 2 \mathrm{H}, 2 \mathrm{NH}), 7 \mathrm{~b}: 7.4-8.4(\mathrm{~m}, 10 \mathrm{H}, \mathrm{Ar}-\mathrm{H}), 8.5(\mathrm{~s}, 1 \mathrm{H}$, chromene-H-4), 9.1, $12.9(2 \mathrm{~s}, 2 \mathrm{H}, 2 \mathrm{NH})$ and 9: $4.0\left(\mathrm{~s}, 3 \mathrm{H}, \mathrm{OCH}_{3}\right), 6.42(\mathrm{~d}, 1 \mathrm{H}, \mathrm{H}-6)$, 7.05-7.52 (m,5H,Ar-H), 8.1(d,1H,H-7), $8.5(\mathrm{~s}, 1 \mathrm{H}, \mathrm{H}-4), 9.5,12.8(2 \mathrm{~s}, 2 \mathrm{H}, 2 \mathrm{NH})$. The mass spectrum of compounds (7b) and (9) afforded the following: Compd. $7 \mathrm{~b} 348$ $(\mathrm{M}+; 4 \%), 313(\mathrm{M}-\mathrm{Cl} ; 100 \%), 222\left(\mathrm{M}-\mathrm{Cl} . \mathrm{C}_{6} \mathrm{H}_{4} \mathrm{NH}, ; 50 \%\right), 167\left[\mathrm{M}-\left(\mathrm{Cl} . \mathrm{C}_{6} \mathrm{H}_{4} \mathrm{NH}+\mathrm{HCN}\right.\right.$ + CO); 10\%], 9: 396 (19.1\%), M+2 (6.8\%), $361(\mathrm{M}-\mathrm{Cl} ; 100 \%), 270\left(\mathrm{M} \mathrm{Cl} . \mathrm{C}_{6} \mathrm{H}_{4} \mathrm{NH}\right.$; $75.2 \%), 227\left[\mathrm{M}-\left(\mathrm{Cl} . \mathrm{C}_{6} \mathrm{H}_{4} \mathrm{NH}\right.\right.$ and $\left.\left.\mathrm{HNCO}\right) ; 29.8 \%\right], 199\left[\mathrm{M}-\left(\mathrm{Cl} . \mathrm{C}_{6} \mathrm{H}_{4} \mathrm{NH}, \mathrm{HNCO}\right.\right.$ and CO); $14.5 \%]$.

\section{Chromene-2-one derivatives 4, 8 and 10:}

Method a: To a solution of $1(0.01 \mathrm{~mol})$ in acetic anhydride $(20 \mathrm{ml})$, the suitable ohydroxyaldehyde $(0.01 \mathrm{~mol})$ and sod. acetate $(0.5 \mathrm{~g})$ were added. The mixture was refluxed for $1 \mathrm{~h}$, the solid product was collected by filtration and recrystallized from the proper solvent to afford $\left(\mathbf{4}^{15}, \mathbf{7}^{16}\right.$ and $\mathbf{1 0}$; Table 1$)$. IR spectrum of compound (4a) showed absorption bands at $3271(\mathrm{NH}), 2916\left(\mathrm{CH}\right.$-aliph.) and $1705 \mathrm{~cm}^{-1}(\mathrm{CO})$. Also, IR of (4b) showed bands at $3264(\mathrm{NH}), 2925$ (CH-aliph.) and 1700, $1663 \mathrm{~cm}^{-1}(\mathrm{CO})$. 8b: 3209(NH), 2923(CH-aliph.) and 1712 (CO). 10: $3425(\mathrm{NH}), 2970$ (CH-aliph) and 1728, 1672 (CO). ${ }^{1} \mathrm{H}-\mathrm{NMR}$ of (4b) revealed multiplet at 7.4-8.0 ppm for aromatic $\mathrm{C}-\mathrm{H}$, singlet at $68.8 \mathrm{ppm}$ assigned to chromene- $\mathrm{H}$ and singlet at $10.6 \mathrm{ppm}$ for $\mathrm{NH}$.

Method b: A Solution of the corresponding iminochromene derivatives 3, 7 and 9 $(0.01 \mathrm{~mol})$ in ethanol $(30 \mathrm{ml})$, hydrochloric acid or acetic acid $(5 \mathrm{ml})$ was refluxed for $1 \mathrm{~h}$. 2-Amino-5-imino-4-oxo-3-(4-tolyl)-3,5-dihydro-4H-chromeno[3,4-c]pyridine-1-carbonitrile (12a), 3-(4-Chlorophenyl)-2-hydroxy-5-imino-4-oxo-3,5dihydro-4H-chromeno[3,4-c]pyridine-1-carbonitrile (12b), 2-Amino-(4-chlorophenyl)-5-imino- 4-oxo3,4a,5,10b-tetrahydro-4H-chromeno[3,4-c]pyridine-1-carbonitrile(13), 2-Amino-3-(4chlorophenyl)-5-imino-4-oxo-3,5-dihydro-4H-benzo[f]chromeno[3,4-c]-1-carbonit-rile 
(14): A mixture of $3 \mathrm{~b}$ or $7 \mathrm{~b}(0.01 \mathrm{~mol})$ and malononitrile or ethyl cyanoacetate $(0.01 \mathrm{~mol})$ and amm.acetate $(0.015)$ in ethanol $(30 \mathrm{ml})$ was refluxed for $3 \mathrm{~h}$. The solid product was collected by filtration and recrystallized to furnish $(\mathbf{1 2 a}, \mathbf{b}, \mathbf{1 3}$ and 14; Table 1). IR spectrum of (12a) showed $\mathrm{NH}, \mathrm{NH}_{2}$ at $3439,3348, \mathrm{CN}$ at 2207 and $\mathrm{CO}$ (amido) at $1650 \mathrm{~cm}^{-1}$. IR spectrum of (12b) showed $\mathrm{OH}, \mathrm{NH}$ broad at 3349, 3176, $\mathrm{CN}$ at 2196 and $\mathrm{CO}$ at $1715 \mathrm{~cm}^{-1}$. 13: $\mathrm{NH}_{2}, \mathrm{NH}$ at 3444, 3348, $\mathrm{CN}$ at 2208 and $\mathrm{CO}$ at $1662 \mathrm{~cm}^{-1}$. 14: showed characteristic absorption bands at 3330, 3214 for $\mathrm{NH}_{2}, \mathrm{NH}$, 2216 for $\mathrm{CN}$ and $1715,1682 \mathrm{~cm}^{-1}$ for $\mathrm{CO} .{ }^{1} \mathrm{H}-\mathrm{NMR}$ spectra in DMSO- $\mathrm{d}_{6}$ revealed the following signals: 12b: 7.3-7.6 $(2 \mathrm{~m}, 8 \mathrm{H}, \mathrm{Ar}-\mathrm{H}), 8.8(\mathrm{~s}, 1 \mathrm{H}, \mathrm{NH}), 9.2(\mathrm{~s}, 1 \mathrm{H}, \mathrm{OH}) .13$ : $4.71(\mathrm{~d}, 1 \mathrm{H}, \mathrm{CH}-4), 4.9(\mathrm{~d}, 1 \mathrm{H}, \mathrm{CH}-3), 7.3-7.6(\mathrm{~m}, 8 \mathrm{H}, \mathrm{Ar}-\mathrm{H}), 7.7\left(\mathrm{~s}, 2 \mathrm{H}, \mathrm{NH}_{2}\right), 8.9(\mathrm{~s}$, $\mathrm{H}, \mathrm{NH})$. 14: 7.6-8.6 (m, 10H, Ar-H), 8.9-9.0 (d, 2H, NH $\left.\mathrm{N}_{2}\right), 9.3(\mathrm{~s}, \mathrm{IH}, \mathrm{NH})$. Mass spectrum of (12a) showed a molecular ion peak at mlz $342(3.2 \%)$ with a base peak at $\mathrm{mlz} 300\left[\mathrm{M}-\left(\mathrm{CH}_{3}+\mathrm{HCN}\right)\right]$, other significant peaks were observed at $\mathrm{m} / \mathrm{z} 272$ [M$\left(\mathrm{CH}_{3}, \mathrm{HCN}\right.$ and $\left.\left.\mathrm{CO}\right) ; 34.0 \%\right], 256\left[\mathrm{M}-\left(\mathrm{CH}_{3} \mathrm{HCN}, \mathrm{CO}\right.\right.$ and $\left.\left.\mathrm{NH}_{2}\right) ; 3.5 \%\right]$ and $230[\mathrm{M}-$ $\left(\mathrm{CH}_{3}, \mathrm{HCN}, \mathrm{CO}, \mathrm{NH}_{2}\right.$, and $\left.\left.\mathrm{CN}\right) ; 4.36 \%\right]$.

\section{2-Amino-5-iminooo-4-oxo-3,5-dihydro-4H-chromeno[3,4-c]pyridine-1-N-(4- chloro-phenyl)carboxamide (16):}

A mixture of 2 iminochromene-3-carboxamide 15 (0.01 mol) and amm.acetate $(0.015 \mathrm{~mol})$ was refluxed for $6 \mathrm{~h}$. The solid product was collected by filtration and crystallized to give (16; Table1). IR spectrum of showed characteristic absorption bands at 3268, 3133 for $\left(\mathrm{NH}_{2}, \mathrm{NH}\right), 2960$ for $\left(\mathrm{CH}\right.$-aliph.) and $1667 \mathrm{~cm}^{-1}$ for $(\mathrm{CO}) .{ }^{1} \mathrm{H}-$ NMR spectrum in DMSO- $\mathrm{d}_{6}$ showed 7.4-7.8 (m, 8H, Ar-H), 9.0(d, 2H, $\left.\mathrm{NH}_{2}\right)$ and 10.3$10.5(\mathrm{br}, 3 \mathrm{H}, 3 \mathrm{NH})$. Its mass spectrum of showed a molecular ion peak at mlz 380 $(9.8 \%)$ with a base peak at $\mathrm{mlz} 253\left(\mathrm{M}-\mathrm{Cl} . \mathrm{C}_{6} \mathrm{H}_{4} \mathrm{NH}_{2}\right)$. Other significant peaks were observed at mlz: 194 (20.9\%), 153 (27\%), 127 (58.5\%) and 55 (73\%). 


\section{REFERENCES}

[1] E. Budzisz, J. G. Wojciechowska, R. Zieba and B. Nawrot, New J. Chem., 26, 1799 (2002).

[2] K. C. Fylaktakidou, D. R. Gautam, D. J. H. Litina, C. A. Kontogiorgis, K. E. Litinas and D. N. Nicolaides, J. Chem. Soc., Per/cm Trans. 1, 3073 (2001).

[3] K. J. M. Salazar, G. E. D. Paredes, L. R. Lluncor, M. C. Max Young, M. J. Kato, Phytochemistry, 66, 573 (2005).

[4] Elnagdi, M.H.; Abdallh, S.O.; Ghoneim, K.M.; Ebied E.M.; and Kassab, K.N.; J. Chem. Research (M) 375 (1997).

[5] Nofal, Z.M.; El-Zahar M.I.; and Abd El-Kariem, S.S.; Molecules, 5, 99 (2000).

[6] Martin, B.R. and Dewey, W.L.; J. Med. Chem., 22, 879 (1979).

[7] Day, W.A. and Lavagnino, E.R.; US pat, 4,152, 450, 01 May (1979); Chem.Abstr.9156822r (1979).

[8] Mat, K.; Stark, P.; and Meister, R.G.; J. Med. Chem., 20, 17 (1977).

[9] El-Gaby, M.S.A.; Ghorab, M.M. and Abdel-Gawad, S.M.; Acta Pharm., 49, 257 (1999).

[10] El-Gaby, M.S.A.; Zahran, M.A. Ismail, M.M.F. and Y.A.Ammar,II Farmaco, 55, 227 (2000).

[11] Sachse, B. and Ertel, H. (Hoeshest A-G) Ger. Offen. 2, 546, 271(C1-A01 Ny120),28 Apr.(1977), Appl.160Ocf (1975); Chem. Abstr. 87, 64057e (1977).

[12] Callaghan, C.N.O. (MRC Irland, Dublin,Ire). Proc..R.Ir.Acad.; Sect. B, 77B, 533 1977).

[13] Carrod, L.P. and Grady, F.D.; Antibiotic and Chemotheraby $3^{\text {rd }}$ ed., Churchill Living- stone, Edinburgh, 477 (1972).

[14] Metwali, M.A.; Hossini, M.S.U.; El-Ablak, F.Z. and Khalil, A.M.; Pharmazie, 47, 336 (1992).

[15] Sammour, A.; Selim, M.I.B. and El-Kady, M.; J. Chem. U. A. R.14, 261 (1971).

[16] El-Sharief, A.M.Sh.; Bedair, A.H.; Ali, F.M.; Murad, A.E. and El-Agrody, A.M.; Egypt J. Chem. 25, 41 (1982); Chem. Abstr. 89, 108949t (1983). 


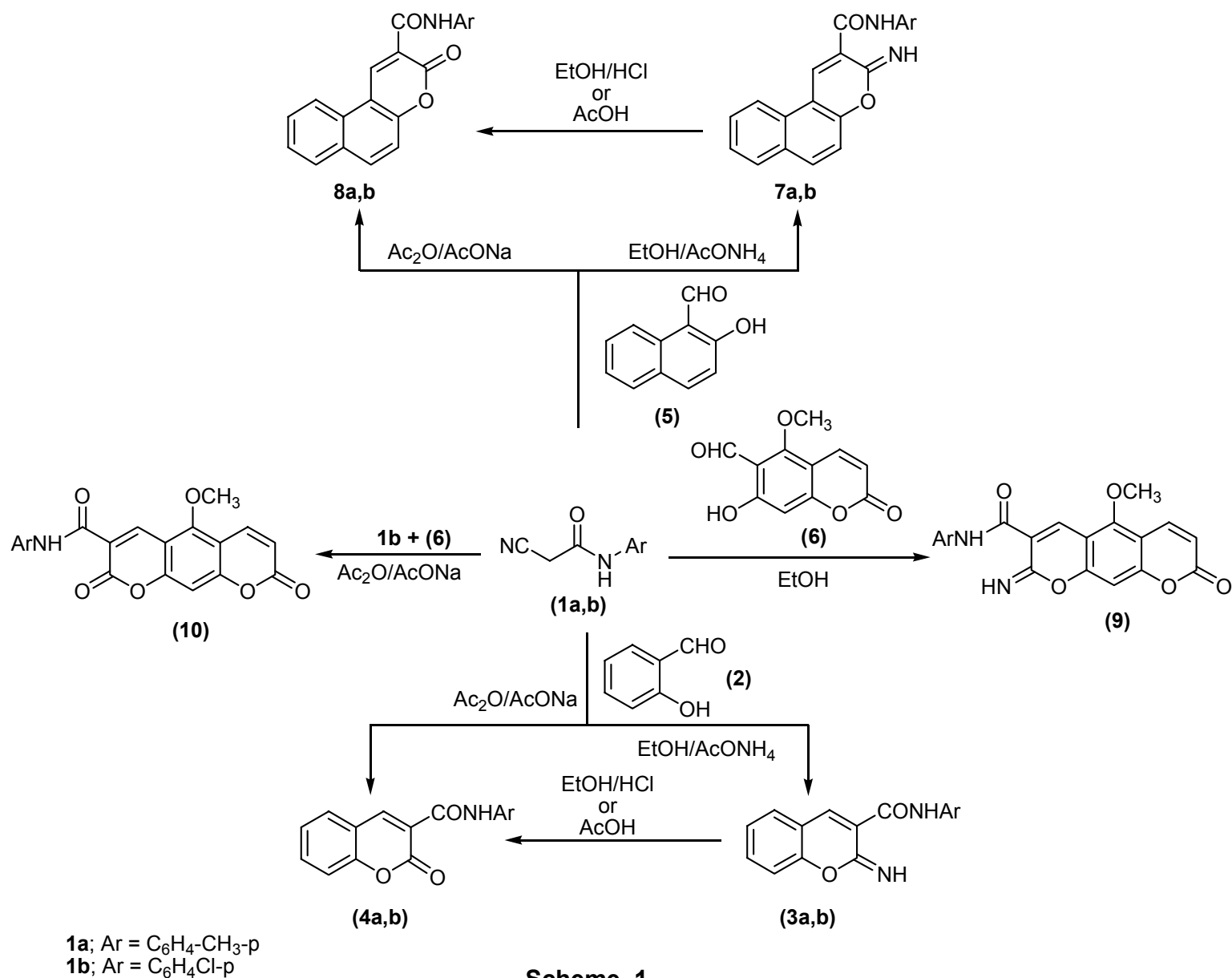

1b; $\mathrm{Ar}=\mathrm{C}_{6} \mathrm{H}_{4} \mathrm{Cl}-\mathrm{p}$

Scheme 1 


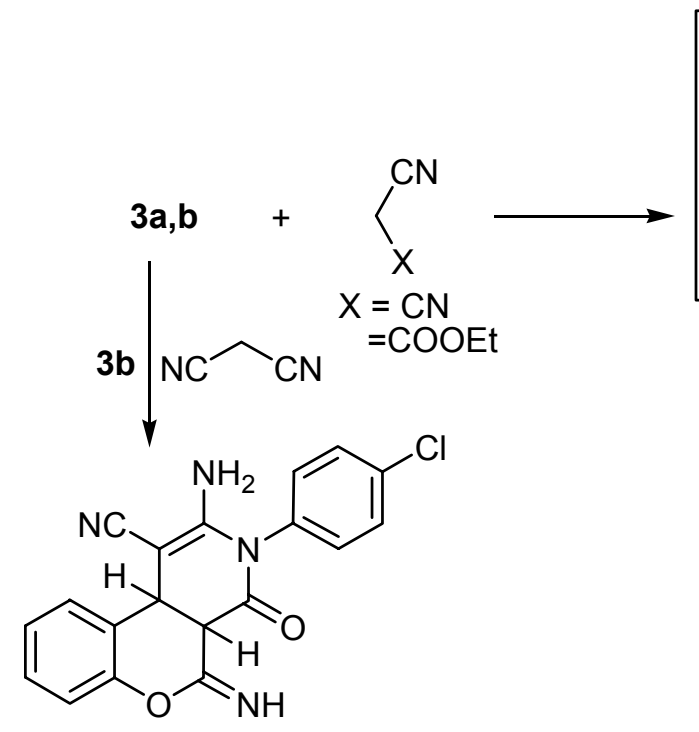

(13)

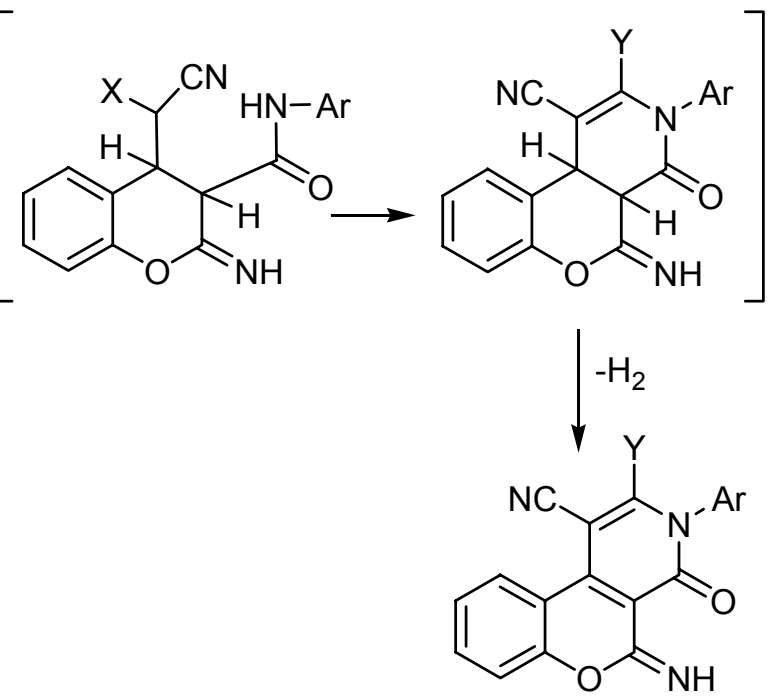

12a, $Y=\mathrm{NH}_{2}, \quad \mathrm{Ar}=\mathrm{C}_{6} \mathrm{H}_{4} \mathrm{CH}_{3}-\mathrm{p}$ 12b, $\mathrm{Y}=\mathrm{OH}, \quad \mathrm{Ar}=\mathrm{C}_{6} \mathrm{H}_{4} \mathrm{Cl}-\mathrm{p}$

\section{Scheme 2}

$7 b+\mathrm{CH}_{2}(\mathrm{CN})_{2} \underset{\mathrm{AcONH}_{4}}{\stackrel{\mathrm{EtOH}}{\longrightarrow}}$<smiles>N#Cc1c(N)n(C2CCCCC2)c(=O)c2c(=N)oc3ccc4ccccc4c3c12</smiles>

14<smiles>N=c1oc2ccccc2cc1C(N)=O</smiles>

15<smiles>CCCCNC(=O)c1c(N)[nH]c(=O)c2c1c(=N)oc1ccccc12</smiles>

16

\section{Scheme 3}


Table 2: Physical data of the prepared compounds.

\begin{tabular}{|c|c|c|c|c|c|c|c|}
\hline \multirow{2}{*}{$\begin{array}{c}\text { Compd } \\
\text { No. }\end{array}$} & \multirow{2}{*}{$\begin{array}{l}\text { M.p. } \\
\left({ }^{\circ} \mathrm{C}\right)\end{array}$} & \multirow{2}{*}{$\begin{array}{c}\text { Solvent } \\
\text { Cryst. }\end{array}$} & \multirow{2}{*}{$\begin{array}{c}\text { Yield } \\
(\%)\end{array}$} & \multirow{2}{*}{$\begin{array}{l}\text { Molecular } \\
\text { formula } \\
\text { (Mol. Wt.) }\end{array}$} & \multicolumn{3}{|c|}{ Elemental analyses } \\
\hline & & & & & $\mathrm{C} \%$ & $\mathrm{H} \%$ & $\mathrm{~N} \%$ \\
\hline \multirow{2}{*}{$3 a$} & \multirow{2}{*}{160} & \multirow{2}{*}{ DMF } & \multirow{2}{*}{80} & $\mathrm{C}_{17} \mathrm{H}_{14} \mathrm{~N}_{2} \mathrm{O}_{3}$ & 73.38 & 5.03 & 10.07 \\
\hline & & & & $(278)$ & 73.40 & 5.10 & 10.00 \\
\hline \multirow{2}{*}{$3 \mathbf{b}$} & \multirow{2}{*}{245} & \multirow{2}{*}{$\mathrm{D}$} & \multirow{2}{*}{75} & $\mathrm{C}_{16} \mathrm{H}_{11} \mathrm{Cl} \mathrm{N}_{2} \mathrm{O}_{2}$ & 64.32 & 3.68 & 9.38 \\
\hline & & & & $(298.5)$ & 64.40 & 3.80 & 9.50 \\
\hline \multirow{2}{*}{$7 a$} & \multirow{2}{*}{235} & \multirow{2}{*}{ D } & \multirow{2}{*}{75} & $\mathrm{C}_{21} \mathrm{H}_{16} \mathrm{~N}_{2} \mathrm{O}_{2}$ & 68.87 & 3.73 & 8.03 \\
\hline & & & & $(328)$ & 68.90 & 3.80 & 8.20 \\
\hline \multirow{2}{*}{$7 b$} & \multirow{2}{*}{217} & \multirow{2}{*}{ DMF } & \multirow{2}{*}{82} & $\mathrm{C}_{20} \mathrm{H}_{13} \mathrm{CIN}_{2} \mathrm{O}$ & 73.65 & 5.45 & 15.16 \\
\hline & & & & $(348.5)$ & 73.74 & 5.40 & 15.22 \\
\hline \multirow{2}{*}{9} & \multirow{2}{*}{310} & \multirow{2}{*}{ DMF } & \multirow{2}{*}{75} & $\mathrm{C}_{20} \mathrm{H}_{13} \mathrm{CIN}_{2} \mathrm{O}_{5}$ & 60.52 & 3.28 & 7.06 \\
\hline & & & & $(396.5)$ & 66.60 & 3.40 & 7.20 \\
\hline \multirow{2}{*}{10} & \multirow{2}{*}{245} & \multirow{2}{*}{$A$} & \multirow{2}{*}{60} & $\mathrm{C}_{20} \mathrm{H}_{12} \mathrm{CINO}_{6}$ & 60.38 & 3.02 & 3.52 \\
\hline & & & & $(397.5)$ & 60.40 & 3.10 & 3.70 \\
\hline \multirow{2}{*}{$11 a$} & \multirow{2}{*}{315} & \multirow{2}{*}{ A } & 54 & $\mathrm{C}_{20} \mathrm{H}_{14} \mathrm{~N}_{4} \mathrm{O}_{2}$ & 70.18 & 3.09 & 16.37 \\
\hline & & & 34 & $(342)$ & 70.20 & 3.20 & 16.60 \\
\hline $11 b$ & 315 & $A$ & 72 & $\mathrm{C}_{19} \mathrm{H}_{10} \mathrm{CIN}_{3} \mathrm{O}_{3}$ & 62.28 & 2.75 & 11.55 \\
\hline & & A & 12 & (363.5) & 62.80 & 2.80 & 11.40 \\
\hline 13 & 290 & D & 66 & $\mathrm{C}_{19} \mathrm{H}_{13} \mathrm{CIN}_{4} \mathrm{O}_{2}$ & 62.55 & 3.57 & 15.36 \\
\hline & & E & & $(364.5)$ & 62.60 & 3.60 & 15.40 \\
\hline 14 & 302 & D & 58 & $\mathrm{C}_{23} \mathrm{H}_{13} \mathrm{CIN}_{4} \mathrm{O}_{2}$ & 66.91 & 3.15 & 13.58 \\
\hline & & & & $(412.5)$ & 66.80 & 3.30 & 13.60 \\
\hline 16 & 315 & DMF & 73 & $\mathrm{C}_{19} \mathrm{H}_{13} \mathrm{NCIN}_{4} \mathrm{O}_{3}$ & 59.92 & 3.42 & 14.72 \\
\hline 10 & & DIVII & 10 & $(380.5)$ & 59.60 & 3.70 & 14.90 \\
\hline
\end{tabular}

$\mathrm{A}=$ Acetic acid, $\mathrm{D}=$ Dioxane, and $\mathrm{DMF}=$ Dimethylformamide 